\title{
BMJ Open Facilitators and barriers to implement nurse-led physical activities for people with dementia in nursing homes: a protocol for a mixed-methods systematic review
}

\author{
Julian Hirt (D) , ${ }^{1,2,3}$ Janine Vetsch, ${ }^{1}$ Steffen Heinrich ${ }^{1}$
}

To cite: Hirt J, Vetsch J, Heinrich S. Facilitators and barriers to implement nurse-led physical activities for people with dementia in nursing homes: a protocol for a mixed-methods systematic review. BMJ Open 2021;11:e056353. doi:10.1136/ bmjopen-2021-056353

- Prepublication history for this paper is available online. To view these files, please visit the journal online (http://dx.doi. org/10.1136/bmjopen-2021056353).

Received 12 August 2021 Accepted 06 December 2021

Check for updates

(C) Author(s) (or their employer(s)) 2021. Re-use permitted under CC BY-NC. No commercial re-use. See rights and permissions. Published by BMJ.

${ }^{1}$ Institute of Applied Nursing Science, Department of Health, Eastern Switzerland University of Applied Sciences, St.Gallen, Switzerland

${ }^{2}$ International Graduate Academy, Institute of Health and Nursing Sciences, Medical Faculty, Martin Luther University Halle Wittenberg, Halle (Saale), Germany

${ }^{3}$ Department of Clinical

Research, University Hospital

Basel, University of Basel, Basel, Switzerland

Correspondence to

Dr Steffen Heinrich;

steffen.heinrich@ost.ch

\section{ABSTRACT}

Introduction Dementia is a major neurocognitive disorder and accounts for a great proportion of nursing home residents. Bodily symptoms such as gait, balance difficulties and muscle weakness are common in people with dementia. Physical activities are important to maintain and promote physical functioning and activities of daily living.

Nurses are a prone stakeholder to deliver physical activity interventions in nursing homes. However, several barriers have been reported for implementing nurse-led physical activity programmes. Also synthesised strategies for sustainable implementation in nursing homes are lacking. We, therefore, aim to synthesise the evidence on physical activity interventions which can be led by nurses and to identify facilitators and barriers of sustainable implementation of physical activity for people with dementia.

Methods and analysis We will conduct a mixedmethods systematic review to (1) describe the characteristics of nurse-led physical activities for people with dementia living in nursing homes and to (2) determine facilitators and barriers for sustainable implementation of nurse-led physical activities for people with dementia in nursing homes. We will search four different electronic databases, do forward and backward citation tracking and will web-search additionally. We will also search through guideline databases. Two reviewers will independently screen the identified studies for their eligibility. Data extraction and critical appraisal of included studies will be performed by one reviewer and doublechecked by another reviewer. We will analyse the data using an integrative mixed-method synthesis and we will summarise the findings narratively.

Ethics and dissemination No ethics approval is needed to perform this systematic review. We will publish our review results as scientific and non-scientific journal articles and we will hold an oral or a poster conference presentation. The review results will inform the development of structured and easy to understand guidance for nurses to initiate tailored physical activities for people with dementia in nursing homes.
Strengths and limitations of this study

- A comprehensive literature search consisting of database searching, citation tracking and supplementary guideline searching with no restrictions to publication date or study design is a strength of this study.

- A mixed-methods systematic synthesis combining evidence from multiple study designs and sources is a strength of this study.

- Involvement of key stakeholders such as nursing experts, nursing managers, stakeholder associations and physiotherapy throughout the review process will allow us to develop recommendations for clinical practice and can be seen as a strength of this study.

- Our study is limited since we rely on published reports of empirical studies and we will exclude studies that do not report physical outcomes in the title and/or abstract.

\section{INTRODUCTION}

Dementia is a degenerative and major neurocognitive disorder accompanied by changes of emotional, social, cognitive and physical behaviour. ${ }^{1}$ The increasing dependence of people with dementia leads to formal and informal care. ${ }^{2}$ Among European countries, the number of people with dementia living in nursing homes highly varies between 1 and 8 per 10 nursing home residents. In Switzerland, people with dementia account for $60 \%$ of nursing home residents. ${ }^{3}$ Bodily symptoms such as limitations with regard to gait, balance and muscle strength are common among people with dementia. ${ }^{4}$ Physical activity can help people with dementia to mitigate these symptoms. ${ }^{5}$

Physical activity is any bodily movement produced by skeletal muscles that requires energy expenditure. Exercises are physical activities and defined as planned, structured, 
repetitive and purposeful actions to improve or maintain one or more components of physical fitness. The resulting effects depend on the intensity and amplitude of the physical activity performance and the person's age. ${ }^{6}$ Physical activity is important to maintain and promote physical functioning. Although there is no single definition, physical functioning describes balance-strength, coordination-strength and muscle-strength capabilities of the body. ${ }^{7}$ A core outcome parameter for physical functioning is fall rate. It has been shown that it can be significantly lowered by physical activity in people with dementia. ${ }^{8}$ Physical activity is also important to maintain and promote activities of daily living, and therefore, independence in people with dementia. ${ }^{79}$ Activities of daily living are characterised by tasks that individuals perform to manage their everyday routines (eg, eating, clothing, walking, toileting). A current Cochrane review concludes that physical exercises improve the ability to perform activities of daily living of people with dementia. ${ }^{5}$

To perform activities of daily living, physical resources are necessary and can be trained by healthcare professionals. ${ }^{10}$ As nurses and nursing and healthcare assistants are the primary carers in nursing homes, they are prone to be a key stakeholder to offer and implement physical activities for people with dementia. However, a systematic and specific synthesis of facilitators and barriers to implement nurse-led physical activities for people with dementia in nursing homes is lacking. ${ }^{11}$ There are global recommendations regarding the implementation of physical activities for community-dwelling people with dementia. ${ }^{12} 13$ Implementation facilitators are participation in preferred physical activities and an individually tailored adoption of exercises for people with dementia. Inhibitors include the unfamiliarity of healthcare providers with the beneficial impact of physical activities, the characteristics of physical activity programmes, and techniques to deliver them tailored to the needs of individuals with dementia. A barrier for the implementation of nurse-led physical activity in nursing homes is the lack of easy to understand and process information. ${ }^{1415}$

To address existing challenges and to develop tailored strategies for successful and sustainable implementation of physical activity interventions in nursing homes, it is important to understand implementation facilitators and barriers and to derive context-sensitive implementation strategies for clinical practice. We, therefore, aim to answer the following review questions:

1. Which nurse-led physical activities are used to maintain and promote physical outcomes of people with dementia living in nursing homes and what are the intervention characteristics?

2. What are facilitators and barriers for sustainable implementation of physical activities for people with dementia in nursing homes?

The results of our review will provide a detailed overview on characteristics of nurse-led physical activities for people with dementia living in nursing homes as well as facilitators and barriers for their context-sensitive implementation based on evidence from a range of study designs and sources.

\section{METHODS AND ANALYSIS}

We will conduct a mixed-methods systematic review to (1) describe the characteristics of nurse-led physical activities for people with dementia living in nursing homes and to (2) determine facilitators and barriers for sustainable implementation of nurse-led physical activities for people with dementia in nursing homes. To structure our review protocol, we used the 2015 statement of the Preferred Reporting Items for Systematic review and Meta-Analysis Protocols. ${ }^{16}$

\section{Eligibility criteria}

To answer our review questions, we are interested in various study designs and study material related to nurse-led physical activities for people with dementia living in nursing homes. We aim at collecting all information on the development, piloting, evaluation and implementation of nurse-led physical activities for people with dementia in nursing homes and to gain a comprehensive overview of intervention characteristics and potential determinants for their implementation. ${ }^{17} 18$ We define nurse-led physical activities as interventions such as exercises, walking, or dancing that are initiated and/or conducted by nurses or nursing or healthcare assistants.

Table 1 provides a detailed overview of inclusion and exclusion criteria related to the two review questions. To answer our first review question, we will include experimental and quasiexperimental studies assessing nurse-led physical activities for people with dementia on physical outcomes in nursing homes published in English, French or German. Physical outcomes such as physical functioning, activities of daily living, or falls need to be reported in the title and/or abstract of a reference. Reports describing the intervention design or the development of nurse-led physical activities will also be included. Studies will not be restricted to type and severity of dementia, gender, age, ethnic background and number of study participants, control type or publication year or publication type. Studies on people with no formal diagnosis of dementia will also be included since there is often no formal medically diagnosis of dementia in long-term care. ${ }^{19}$ We will exclude studies with participants reporting mild cognitive impairment and mixed samples with less than $75 \%$ of people with dementia (if the results are not reported separately). We will include studies that refer to the nursing home setting. We define nursing home as a setting where people (can) receive 24-hour professional nursing care as there exists variations and country-specific adaptations. Accordingly, we will also include studies from care homes, homes for the aged, and residential aged care facilities. ${ }^{20}$ Studies conducted in other institutionalised care facilities such as assisted living facilities or non-institutionalised care will be excluded. We will also exclude mixed settings with less than $75 \%$ of people with dementia from nursing homes (if the results are not reported separately). Further, abstract only publications, editorials, commentaries and letters will be excluded. 
Table 1 Inclusion and exclusion criteria

\begin{tabular}{|c|c|c|c|}
\hline Domain & Inclusion criteria & Exclusion criteria & Review question \\
\hline \multirow[t]{2}{*}{ Population } & $\begin{array}{l}\text { People with all types of dementia with no } \\
\text { restriction to severity, gender, age, ethnic } \\
\text { background, diagnostic procedures and no of } \\
\text { study participants }\end{array}$ & $\begin{array}{l}\text { People with mild cognitive impairment } \\
\text { and mixed samples with less than } 75 \% \\
\text { people with dementia (if the results are not } \\
\text { reported separately) }\end{array}$ & $1 / 2$ \\
\hline & $\begin{array}{l}\text { Health professionals and/or family members } \\
\text { of people with dementia and/or nursing home } \\
\text { managers with no restriction to number of } \\
\text { study participants (if implementation facilitators } \\
\text { and barriers are reported belonging to a } \\
\text { specific nurse-led physical activity intervention } \\
\text { for people with dementia in nursing homes) }\end{array}$ & - & 2 \\
\hline Setting & $\begin{array}{l}\text { Nursing homes and other institutionalised care } \\
\text { settings (eg,care homes, homes for the aged, } \\
\text { residential-aged care facilities) }\end{array}$ & $\begin{array}{l}\text { Other institutionalised care facilities (eg, } \\
\text { assisted living facilities, day care) or non- } \\
\text { institutionalised care }\end{array}$ & $1 / 2$ \\
\hline \multirow[t]{3}{*}{ Study aim } & $\begin{array}{l}\text { Assessing the effects/effectiveness/efficacy/ } \\
\text { feasibility of nurse-led physical activities }\end{array}$ & - & 1 \\
\hline & $\begin{array}{l}\text { Description of intervention design and } \\
\text { modelling or developing nurse-led physical } \\
\text { activities }\end{array}$ & - & 1 \\
\hline & $\begin{array}{l}\text { Determining facilitators and/or barriers to } \\
\text { implement nurse-led physical activities }\end{array}$ & - & 2 \\
\hline Control & Every and no control type & - & 1 \\
\hline Outcome & $\begin{array}{l}\text { Physical outcomes (eg, physical functioning } \\
\text { (lower and upper body), activities of daily living, } \\
\text { falls) reported in the title and/or abstract of a } \\
\text { reference }\end{array}$ & - & 1 \\
\hline
\end{tabular}

\begin{tabular}{llll}
\hline Study design & $\begin{array}{l}\text { Experimental and quasi-experimental studies } \\
\text { (controlled clinical trials, before-and-after } \\
\text { study) }\end{array}$ & Reviews \\
& $\begin{array}{l}\text { All primary study designs (eg, observational } \\
\text { studies, interventional studies, qualitative } \\
\text { studies, mixed-methods studies, process } \\
\text { evaluations) }\end{array}$ & Reviews \\
\hline $\begin{array}{l}\text { No restriction } \\
\text { Publication year }\end{array}$ & $\begin{array}{l}\text { English, French, German } \\
\text { language }\end{array}$ & - & - \\
\hline $\begin{array}{l}\text { Publication type } \\
\text { Any published reports of empirical studies } \\
\text { (eg, journal articles, conference proceedings, } \\
\text { preprints) }\end{array}$ & $\begin{array}{l}\text { Abstract-only publications, editorials, } \\
\text { commentaries, letters, study protocols, } \\
\text { theses, registry entries, books }\end{array}$ & $1 / 2$ \\
\hline $\begin{array}{l}\text { Online and offline material associated with the } \\
\text { intervention }\end{array}$ & - & $1 / 2$ \\
\hline
\end{tabular}

To answer our second review question, it requires evidence from studies determining facilitators and/or barriers to implement nurse-led physical activities in nursing homes reported belonging to a specific nurse-led physical activity intervention for people with dementia (eg, acceptance, feasibility, resources). This can be reached by using various study designs such as observational studies, interventional studies, qualitative studies, mixed-methods studies and process evaluations. No study design will explicitly be excluded. As (mixed) study populations, we will include studies with people with dementia, health professionals, family members of people with dementia and nursing home managers with no restriction to number of study participants that report implementation facilitators and barriers of nurse-led physical activity 
interventions for people with dementia in nursing homes. Inclusion criteria concerning publication year, language, and study type will be equal to the first review question.

\section{Information sources}

Database searching and supplementary search methods

We will perform a comprehensive literature search combining electronic database searching with supplementary search methods. ${ }^{21}$ We will search MEDLINE via PubMed, CINAHL, Cochrane Central Register of Controlled Trials via Cochrane Library, and Web of Science Core Collection. As a supplementary search method, we will conduct forward and backward citation tracking of included studies using Scopus. We selected MEDLINE/ PubMed, CINAHL, Web of Science Core Collection, and citation tracking since it is an optimal combination of search methods in dementia care research. ${ }^{22}$ To identify additional studies that are not indexed in the above listed databases and to search for study material related to nurse-led physical activity interventions, we will conduct web searching using Google and Google Scholar. To identify pertinent clinical practice guidelines, we will search the GIN international guideline library and registry of guidelines in development, ${ }^{23}$ FIT-nursing care, ${ }^{24}$ as well as guidelines provided by the Association of the Scientific Medical Societies in Germany, ${ }^{25}$ and the German ZQP Foundation. ${ }^{26}$

\section{Study teams' request}

In case of missing information on intervention characteristics or incomplete outcome data, we will reach out to study teams and request the missing information via mail. Two reminders will be sent on a weekly basis. In case of no answer by study teams after 3 weeks, we will declare the information as missing.

\section{Search strategy}

We will use database-specific search strategies based on controlled vocabulary (ie, MeSH for MEDLINE, Subject Headings for CINAHL) and unspecified free text terms. The search strategies will be developed and peer reviewed within the review group using PRESS (Peer Review of Electronic Search Strategies). ${ }^{27}$ We will use three search components: dementia, physical activity and nursing homes. The search strings will be adapted from available reviews on dementia, ${ }^{28}$ physical activity ${ }^{5}$ and nursing homes $^{29}$ that are transparently reported. Search terms and synonyms will be complemented by preliminary searches in MEDLINE/PubMed (ie, title and abstracts of eligible studies) and the experience of the review team members. We will search unspecified free text terms in the title, abstract and keywords field, if available.

Box 1 shows the final search string for MEDLINE/ PubMed. We will manually translate the search string considering database-specific functions and techniques to use it in the other databases. For databases that do not provide systematic search engines, we will use highly relevant search terms and combinations to specifically
Box 1 Search string for Medline via PubMed (searching all fields)

("DEMENT*"[TIAB] OR "ALZHEIMER*"[TIAB] OR "MAJOR NEUROCOGNITIVE DISORDER*"[TIAB] OR "DEMENTIA"[MESH])

AND

("Nursing Homes" [MESH] OR "Homes for the Aged"[Mesh] OR "LongTerm Care" [Mesh] OR "Iongterm care" [tiab] OR "Iong-term care" [tiab] OR "nursing home*"[tiab] OR "Care home*"[tiab] OR "homes for the aged" [tiab] OR "ELDERLY CARE" OR (resident*[tiab] AND (longterm*[tiab] OR long-term*[tiab] OR institut*[tiab])))

AND

(Exercise[MESH] OR "Exercise Therapy"[MESH] OR "Exercise Test"[MESH] OR DANCING[MESH] OR Swimming[MESH] OR "Fitness Centers"[MESH] OR Walking[MESH] OR Yoga[MESH] OR "Tai Ji"[MESH] OR "Physical Fitness" [MESH] OR "Exercise Movement Techniques"[MESH] OR "Physical Exertion" [MESH] OR exercis*[TIAB] $O R$ "physical activit*"[TIAB] OR cycling[TIAB] OR swim* [TIAB] OR gymn*[TIAB] OR walk*[TIAB] or treadmill[TIAB] OR HIKING[TIAB] OR danc*[TIAB] OR yoga*[TIAB] OR "tai chi" [TIAB] OR "TAl Jl"[TIAB] OR fitness $\left.{ }^{*}[T I A B]\right)$

retrieve eligible articles. The search strategies for all databases and search engines will be reported in full detail as part of the final review report.

\section{Study records}

Data management

A bibliography management tool will be used to manage the number of reference retrievals and to detect duplicates (ie, database search, supplementary search methods and web searching). Throughout the study selection process, we will use Rayyan to ensure blinded study selection. ${ }^{30}$

\section{Selection process}

Two reviewers will independently assess titles, abstracts, and, if eligible, full texts for inclusion and exclusion. total of $5 \%-10 \%$ of titles and abstracts as well as full texts will be screened independently by all reviewers. This calibration module will help to understand and apply our eligibility criteria. Following the screening process of the two phases (titles and abstracts, full texts), conflicting assessments will be discussed within the screening team and with a third member of the review team-if necessary.

\section{Data collection process}

Data extraction sheets will be piloted and therefore preliminary applied using ten percent of the included studies. Subsequently, one reviewer will extract data and a second reviewer will double-check data extraction for accuracy. Extraction results will be discussed between the person extracting the data and the person doublechecking the data extraction and with a third member of the review team-if necessary.

\section{Data items}

We will perform data extraction according to the review questions using tabulated templates. To answer our first review question on interventions and their characteristics, 
we will extract data on study design, country, population (ie, age, gender and type and severity of dementia) and setting (eg, nursing home, care home), intervention and control intervention(s), outcome(s) and outcome measurement(s), and results related to physical functioning of people with dementia. To provide a detailed overview of intervention and control intervention characteristics and their theoretical background, we will apply the Template for Intervention Description and Replication (TIDieR). ${ }^{18}$ TIDieR contains items on intervention details such as format, duration, frequency, dose, place and material used, and information on the background and expertise of interventionists which is helpful to describe intervention characteristics.

To answer our second review question on implementation facilitators and barriers, we will use the template provided by the Integrated Sustainability Framework ${ }^{31}$ that was previously used in another systematic review. ${ }^{32}$ The Integrated Sustainability Framework focuses on long-term implementation of health interventions and contains items that are related to factors determining sustainability among clinical contexts and interventions. ${ }^{31}$ It covers contextual factors (eg, management and leadership, sociopolitical context), intervention processes (eg, training and support, fidelity, adaption), characteristics of the interventionists and population (eg, attitudes, motivation) and characteristics of the intervention (eg, adaptability, benefits, costs).

\section{Critical appraisal of individual studies}

To critically appraise the quality of individual studies, we will use the Mixed Methods Appraisal Tool (MMAT). ${ }^{33}$ MMAT can be applied to multiple study designs such as interventional, observational, qualitative and mixedmethods studies. The assessment contains overall quality criteria (eg, if a study contains a clear research question/ aim) as well as basic quality criteria related to each of the study designs mentioned above (eg, if findings were adequately derived from the data in qualitative studies, complete outcome data in randomised controlled trials, appropriateness of measurement(s) in observational studies). One reviewer will conduct critical appraisal and a second reviewer will double-check it. We will discuss appraisal results between the person appraising the studies and the person double-checking the critical appraisal and with a third member of the review team-if necessary.

\section{Data synthesis}

For data synthesis, we will use tabulated data extractions and apply an integrative mixed-methods synthesis of the evidence coming from multiple study designs and source material. ${ }^{34}$ We will write a narrative summary for intervention characteristics (addressing the first review question) and for implementation facilitators and barriers (addressing the second review question) of nurse-led physical activities for people with dementia in nursing homes.

\section{Patient and public involvement}

The results of our review will inform the development of best-practice nurse-led physical activity for people with dementia in (Swiss) nursing homes considering contextsensitive implementation strategies. Therefore, we will recruit an expert panel. Six to ten persons will be part of the local expert panel representing nursing home managers, nursing experts, stakeholder associations and physical therapists. The collaboration with the expert panel will cover two main tasks. First, our review questions will be complemented by subquestions related to the specific interests of the experts and the Swiss nursing home context. Second, the results of our review will be presented to and discussed with the experts. By merging the results of our review with the perspectives of the experts, we aim at presenting a detailed and consented guidance of physical activities and expert view regarding successful and sustainable context-sensitive implementation factors for clinical practice.

The meetings between the review team and expert panel will take place in a conference room at the Eastern Switzerland University of Applied Sciences in St.Gallen, Switzerland. If restrictions due to COVID-19 will take place, the expert panel will be conducted virtually using a web-based teleconference tool. The expert panel will receive written information and material proceeding before each meeting.

\section{Ethics and dissemination}

Taking part in the expert panel will indicate consent on participation. There will be no mandatory participation and experts may leave the process at any time once experts consented to participate. Experts will not receive an incentive for taking part in the expert panel. With regard to the Swiss Human Research Act, our expert panel does not apply to research on human diseases and the human body. ${ }^{35}$ Therefore, no ethical approval is needed.

We will publish our review results as both scientific and non-scientific journal articles and an oral or a poster conference presentation will be held.

The review results will inform the development of structured and easy to understand guidance for nurses to initiate tailored physical activities for people with dementia in nursing homes. Nursing staff with various degrees from assistants or aides to postgraduates will be able to use the guidance in their daily practice. This will be achieved by different sections with tailored information for nurses holding various skill levels. The guidance will be used as a central dissemination tool to facilitate the implementation of nurse-led physical activities for people with dementia in the (Swiss) nursing homes.

\section{Study status}

We will start our search in September 2021 and expect to complete our review in December 2022.

Current study status: preliminary searches: no; piloting of the study selection process: no; formal screening 
of search results against eligibility criteria: no; data extraction: no; data analysis: no.

Contributors $\mathrm{JH}$ and SH were involved in developing the idea for this review. $\mathrm{JH}$, $\mathrm{JV}$ and SH made substantial contributions to the conception and design of the work. $\mathrm{JH}$ and SH drafted the manuscript which was critically reviewed by $\mathrm{JH}, \mathrm{JV}$ and $\mathrm{SH}$ for important intellectual content. JH, JV and $\mathrm{SH}$ gave their final approval of the manuscript version to be published and agreed to be accountable for all aspects of the manuscript.

Funding This review is part of an overarching project focusing on dementia and the ageing society (AGE-INT) funded by the Swiss State Secretariat for Education, Research and Innovation (SERI).

Disclaimer The funder had no influence on the design and the results of this research.

\section{Competing interests None declared.}

Patient and public involvement Patients and/or the public were involved in the design, or conduct, or reporting, or dissemination plans of this research. Refer to the Methods section for further details.

Patient consent for publication Not applicable.

Provenance and peer review Not commissioned; externally peer reviewed.

Open access This is an open access article distributed in accordance with the Creative Commons Attribution Non Commercial (CC BY-NC 4.0) license, which permits others to distribute, remix, adapt, build upon this work non-commercially, and license their derivative works on different terms, provided the original work is properly cited, appropriate credit is given, any changes made indicated, and the use is non-commercial. See: http://creativecommons.org/licenses/by-nc/4.0/.

\section{ORCID iD}

Julian Hirt http://orcid.org/0000-0001-6589-3936

\section{REFERENCES}

1 World Health Organization. International statistical classification of diseases and related health problems 10th revision, 2016. Available: https://icd.who.int/browse10/2016/en\#/F00 [Accessed 21 Jul 2021].

2 Prince M, Prina M, Guerchet M. World Alzheimer report 2013: journey of caring. An analysis of long-term care for dementia, 2013. Available: https://www.alz.co.uk/research/WorldAlzheimerRe port2013.pdf [Accessed 19 Mar 2020].

3 Alzheimer Europe. Dementia in Europe Yearbook 2017: standards for residential care facilities in Europe, 2017. Available: https://www. alzheimer-europe.org/Policy/Country-comparisons/2017-Standardsfor-Residential-Care-Facilities/Introduction/Prevalence-andregulation\#fragment1 [Accessed 21 Jul 2021].

4 Beauchet O, Allali G, Berrut G, et al. Gait analysis in demented subjects: interests and perspectives. Neuropsychiatr Dis Treat 2008;4:155-60.

5 Forbes D, Forbes SC, Blake CM. Exercise programs for people with dementia. Cochrane Database Syst Rev 2015;4:CD006489.

6 WHO. Guidelines on physical activity and sedentary behaviour: web annex: evidence profiles, 2020. Available: https://apps. who int/iris/bitstream/handle/10665/336657/9789240015111-eng.pdf? isAllowed $=\mathrm{y} \&$ sequence $=1$

7 Lam FMH, Huang M-Z, Liao L-R, et al. Physical exercise improves strength, balance, mobility, and endurance in people with cognitive impairment and dementia: a systematic review. J Physiother 2018;64:4-15

8 Gulka HJ, Patel V, Arora T, et al. Efficacy and generalizability of falls prevention interventions in nursing homes: a systematic review and meta-analysis. J Am Med Dir Assoc 2020;21:1024-35.

9 Campbell E, Petermann-Rocha F, Welsh P, et al. The effect of exercise on quality of life and activities of daily life in frail older adults: a systematic review of randomised control trials. Exp Gerontol 2021;147:111287.

10 Roberts CE, Phillips LH, Cooper CL, et al. Effect of different types of physical activity on activities of daily living in older adults: systematic review and meta-analysis. J Aging Phys Act 2017;25:653-70.

11 Karrer $M$, Hirt J, Zeller $A$, et al. What hinders and facilitates the implementation of nurse-led interventions in dementia care? A scoping review. BMC Geriatr 2020;20:127.
12 van Alphen HJM, Hortobágyi T, van Heuvelen MJG. Barriers, motivators, and facilitators of physical activity in dementia patients: a systematic review. Arch Gerontol Geriatr 2016;66:109-18.

13 Karssemeijer EGA, de Klijn FH, Bossers WJR, et al. Ranking barriers, motivators, and facilitators to promote physical activity participation of persons with dementia: an explorative study. J Geriatr Phys Ther 2020;43:71-81.

14 Baert V, Gorus E, Calleeuw K, et al. An administrator's perspective on the organization of physical activity for older adults in long-term care facilities. J Am Med Dir Assoc 2016;17:75-84.

15 Baert V, Gorus E, Guldemont N, et al. Physiotherapists' perceived motivators and barriers for organizing physical activity for older long-term care facility residents. J Am Med Dir Assoc 2015;16:371-9.

16 Moher D, Shamseer L, Clarke M, et al. Preferred reporting items for systematic review and meta-analysis protocols (PRISMA-P) 2015 statement. Syst Rev 2015;4.

17 Craig P, Petticrew M. Developing and evaluating complex interventions: reflections on the 2008 MRC guidance. Int J Nurs Stud 2013;50:585-7.

18 Hoffmann TC, Glasziou PP, Boutron I, et al. Better reporting of interventions: template for intervention description and replication (TIDieR) checklist and guide. BMJ 2014;348:g1687.

19 Nygaard HA, Ruths S. Missing the diagnosis: senile dementia in patients admitted to nursing homes. Scand J Prim Health Care 2003;21:148-52.

20 Berg V. What is the difference between a care home and a nursing home? 2021. Available: https://www.carehome.co.uk/advice/ what-is-the-difference-between-a-care-home-and-a-nursing-home [Accessed 04 Aug 2021]

21 Cooper C, Booth A, Varley-Campbell J, et al. Defining the process to literature searching in systematic reviews: a literature review of guidance and supporting studies. BMC Med Res Methodol 2018;18:85

22 Hirt J, Bergmann J, Karrer M. Overlaps of multiple database retrieval and citation tracking in dementia care research: a methodological study. J Med Libr Assoc 2021;109:275-85.

23 Guidelines International Network (GIN). Gin international guideline library and registry of guidelines in development, 2021. Available: https://g-i-n.net/international-guidelines-library/ [Accessed $20 \mathrm{Jul}$ 2021].

24 Eastern Switzerland University of Applied Sciences. FIT-Nursing care, 2021. Available: https://www.fit-care.ch/ [Accessed 20 Jul 2021].

25 Association of the Scientific Medical Societies in Germany (AWMF). Guidelines, 2021. Available: https://www.awmf.org/en/clinicalpractice-guidelines/search-for-guidelines.html [Accessed $21 \mathrm{Jul}$ 2021].

26 ZQP Foundation. Nursing guidelines and standards, 2021. Available: https://lls.zqp.de/ [Accessed $20 \mathrm{Jul}$ 2021].

27 McGowan J, Sampson M, Salzwedel DM, et al. PRESS Peer Review of Electronic Search Strategies: 2015 Guideline Statement. J Clin Epidemiol 2016;75:40-6.

28 Hirt J, Ballhausen N, Hering A, et al. Social robot interventions for people with dementia: a systematic review on effects and quality of reporting. J Alzheimers Dis 2021;79:773-92.

29 Groot Kormelinck CM, Janus SIM, Smalbrugge M, et al. Systematic review on barriers and facilitators of complex interventions for residents with dementia in long-term care. Int Psychogeriatr 2021:33:873-89.

30 Ouzzani M, Hammady H, Fedorowicz Z, et al. Rayyan - a web and mobile app for systematic reviews. Syst Rev 2016;5:210.

31 Shelton RC, Cooper BR, Stirman SW. The sustainability of evidencebased interventions and practices in public health and health care. Annu Rev Public Health 2018;39:55-76.

32 Shoesmith A, Hall A, Wolfenden L, et al. Barriers and facilitators influencing the sustainment of health behaviour interventions in schools and childcare services: a systematic review. Implementation Sci 2021;16:62.

33 Hong QN, Pluye P, bregues S F. Mixed methods appraisal too (MMAT) version 2018: user guide, 2019. Available: http://mixedmet hodsappraisaltoolpublic.pbworks.com/w/file/fetch/127916259/ MMAT_2018_criteria-manual_2018-08-01_ENG.pdf [Accessed 20 Jul 2021].

34 Heyvaert M, Hannes K, Onghena P. Using mixed methods research synthesis for literature reviews. London: SAGE Publications, 2017.

35 Gloy V, McLennan S, Rinderknecht M, et al. Uncertainties about the need for ethics approval in Switzerland: a mixed-methods study. Swiss Med Wkly 2020;150:w20318. 